\title{
A COUNTEREXAMPLE TO A RESULT CONCERNING CONTROLLED APPROXIMATION ${ }^{1}$
}

\author{
RONG-QING JIA
}

\begin{abstract}
A result of Strang and Fix states that if the order of controlled approximation from a collection of locally supported elements is $k$, then there is a linear combination $\Omega$ of those elements and their translates such that any polynomial of degree less than $k$ can be reproduced by $\Omega$ and its translates. This paper gives a counterexample to their result.
\end{abstract}

This paper concerns the so-called controlled approximation. This concept was introduced by Strang $[\mathbf{S}]$ in 1970. In that paper, Strang described a systematic approach to the choice of trial functions used in finite element analysis. (Also see [FS] for some related results.) To describe his approach, we first introduce some notation. As usual, we mean by $W^{k, p}\left(\mathbf{R}^{n}\right)$ the usual Sobolev space with norm

$$
\|u\|_{k, p}:=\sum_{|\alpha| \leq k}\left\|D^{\alpha} u\right\|_{L^{p}} .
$$

(Here we use standard multi-index notation.) The seminorm $|\cdot|_{k, p}$ on $W^{k, p}\left(\mathbf{R}^{n}\right)$ is defined to be

$$
|u|_{k, p}:=\sum_{|\alpha|=k}\left\|D^{\alpha} u\right\|_{L^{p}}
$$

When $k=0,|\cdot|_{k, p}$ is a norm, and we write $\|u\|_{p}:=|u|_{0, p}=\|u\|_{L^{p}}$. By $W_{c}^{k, p}\left(\mathbf{R}^{n}\right)$ we denote the space of all functions in $W^{k, p}\left(\mathbf{R}^{n}\right)$ that have compact support. By $l^{p}\left(\mathbf{Z}^{n}\right)$ we mean the space of the mappings $w$ from $\mathbf{Z}^{n}$ to $\mathbf{R}$ which satisfy

$$
\|w\|_{p}:=\left(\sum_{j}|w(j)|^{p}\right)^{1 / p}<\infty .
$$

Strang's approach can be described as follows: Choose one or more functions $\phi_{1}, \ldots, \phi_{N}$ in $W_{c}^{k, 2}\left(R^{n}\right)$, rescale the independent variables, obtaining $h^{-n / 2} \phi_{i}(\cdot / h)$ with $h>0(i=1, \ldots, N)$, and translate the functions just constructed, replacing $x$ by $x-j h$. Thus we have a family of trial functions:

$$
\phi_{i, j}^{h}(x):=h^{-n / 2} \phi_{i}(x / h-j), \quad i=1, \ldots, N, j \in \mathbf{Z}^{n} .
$$

When $h=1$, we omit the superscript $h$. Note that the coefficient $h^{-n / 2}$ normalizes $\phi_{i, j}^{h}$ in the sense that $\left\|\phi_{i, j}^{h}\right\|_{2}=\left\|\phi_{i}\right\|_{2}$.

A principal question in finite element analysis is the degree of approximation which can be achieved by the span of $\phi_{i, j}^{h}$. Concerning this problem, Strang and Fix state the following result (see [SF, Theorem II]):

Received by the editors September 24, 1984 and, in revised form July 29, 1985.

1980 Mathematics Subject Classification. Primary 41A29.

${ }^{1}$ Sponsored by the United States Army under Contract No. DAAG29-80-C-0041. This material is based upon work supported by the National Science Foundation under Grant No. MCS-8210950. 
THEOREM A. Suppose $\phi_{1}, \ldots, \phi_{N}$ are in $W_{c}^{k, 2}\left(\mathbf{R}^{n}\right)$. Then the following conditions are equivalent:

(i) ${ }_{k}$ For each $u$ in $W^{k+1,2}$ and $h>0$, there are weights $w_{i, j}^{h}$ such that

$$
\left|u-\sum_{i, j} w_{i, j}^{h} \phi_{i, j}^{h}\right|_{s, 2} \leq \text { const }_{s} h^{k+1-s}|u|_{k+1,2} \quad \text { for } s \leq k,
$$

$$
\left\|w_{i}^{h}\right\|_{2} \leq \text { const }\|u\|_{2} .
$$

(Here $w_{i}^{h}$ is the mapping $j \rightarrow w_{i, j}^{h}, j \in \mathbf{Z}^{n}$.)

(ii) ${ }_{k}$ There is a finite linear combination $\Omega$ of $\phi_{1}, \ldots, \phi_{N}$ and their translates $\phi_{i, j}$ such that

$$
\begin{aligned}
& \text { for }|\alpha| \leq k, \sum_{j \in \mathbf{Z}^{n}} j^{\alpha} \Omega(x-j) \text { is a polynomial in } x_{1}, \ldots, x_{n} \text { with } \\
& \text { leading term } x^{\alpha} .
\end{aligned}
$$

If a collection $\Phi=\left\{\phi_{1}, \ldots, \phi_{N}\right\}$ satisfies (i) ${ }_{k-1}$ but not (i) ${ }_{k}$, then we say that $\Phi$ has controlled approximation order $k$. Note that (2) constrains the coefficients $w_{i, j}^{h}$. This is the reason why $[\mathbf{S}]$ calls such approximation controlled.

The implication of $(\mathrm{i})_{k}$ from (ii) ${ }_{k}$ is in no doubt. This has been proved by several authors, in particular, by $[\mathbf{S F}]$ (see the references cited by $[\mathbf{S}]$ and $\left[\mathbf{F S}_{2}\right]$ ). But, the other direction, i.e., the implication of (ii) ${ }_{k}$ from $(\mathrm{i})_{k}$ has been proved only for $N=1$ by [SF]; the proof for the case $N>1$ is not discussed in [SF].

Recently, the rapid development of the theory of multivariate spline functions has given a new impetus to the controlled approximation problem. In [DM], Theorem A was cited to determine the controlled approximation order from certain bivariate box splines. In order to advance the theory of controlled approximation, it becomes important to answer the question whether (i) $k$ implies (ii) $k$.

As it turns out, (i) $)_{k}$ does not imply (ii) $)_{k}$ in general. We shall give a counterexample in the following. In our example, $n=2, N=4$, and $\phi_{1}, \ldots, \phi_{4}$ are splines with compact support, which are constructed from box splines. Thus we have to recall box splines. Before doing so, we need to introduce more notation. The vectors $e_{i}(i=1,2,3)$ in $\mathbf{R}^{2}$ are given by

$$
e_{1}=(1,0), \quad e_{2}=(0,1) \text { and } e_{3}=(1,1) .
$$

The difference operators $\nabla_{i}$ are given by the rule

$$
\nabla_{i} f=f-f\left(\cdot-e_{i}\right), \quad \text { for } f: Z^{2} \rightarrow \mathbf{R} .
$$

The differential operators $D_{i}$ are given by

$$
D_{i}=\frac{\partial}{\partial x_{i}} \quad(i=1,2) \quad \text { and } \quad D_{3}=D_{1}+D_{2} .
$$

Moreover, we denote by $\pi_{k}$ the space of all polynomials in two variables of total degree no more than $k$. Let us recall the definition of box splines from [BH]. For a sequence $\Xi=\left(\xi_{i}\right)_{1}^{n}$ of vectors in $\mathbf{R}^{n}$, the box spline $M_{\Xi}$ is defined to be the distribution given by

$$
\left\langle M_{\Xi}, \phi\right\rangle=\int_{[0,1]^{n}} \phi\left(\sum_{i=1}^{n} \lambda(i) \xi_{i}\right) d \lambda
$$


for any $C^{\infty}$-locally supported function $\phi$. If $\Xi$ consists of $e_{1}, e_{2}$ and $e_{3}$, repeated $r, s$ and $t$ times, respectively, then we write $M_{r, s, t}$ instead of $M_{\Xi}$. In particular

$$
M_{r, s, 0}\left(x_{1}, x_{2}\right)=M_{r}\left(x_{1}\right) M_{s}\left(x_{2}\right) .
$$

Here $M_{r}$ is the usual (univariant) $B$-spline of order $r$ at the knot sequence $0,1, \ldots, r$ :

$$
M_{r}(x)=[0,1, \ldots, r](\cdot-x)_{+}^{r-1},
$$

where $\left[\rho_{0}, \rho_{1}, \ldots, \rho_{r}\right] \mathcal{F}$ means the divided difference of $f$ at points $\rho_{0}, \rho_{1}, \ldots, \rho_{r}$.

Now we can formulate our collection $\Phi=\left\{\phi_{1}, \phi_{2}, \phi_{3}, \phi_{4}\right\}$. Our candidates are $M_{2,0,1}, M_{2,1,0}, M_{0,2,1}$ and $M_{1,2,0}$. But we want to modify them to suit our purpose. First, for a technical reason which will be clear later, we want to shift those splines. Thus we define the shift operator $\tau$ by

$$
\tau f:=f\left(\cdot+e_{3}\right) .
$$

Second, and more importantly, those splines are not in the space $W^{1,2}$. So we have to smooth them. This can be done by convolving them with a sufficiently smooth function. We should be careful in doing so, however, because we want to keep $\Phi$ from satisfying (ii) ${ }_{1}$. Thus we introduce the convolution operator $\sigma$ as follows:

$$
\begin{aligned}
\sigma f & :=\int f\left(x_{1}-t, x_{2}-t\right) 2 M_{1}\left(2 t+\frac{1}{2}\right) d t \\
& =2 \int_{-1 / 4}^{1 / 4} f\left(x_{1}-t, x_{2}-t\right) d t .
\end{aligned}
$$

Note that $\sigma$ and $\tau$ commute with each other. Let

$$
\begin{array}{ll}
\phi_{1}:=\sigma \tau\left(M_{2,0,1}\right), & \phi_{2}:=\sigma \tau\left(M_{2,1,0}\right), \\
\phi_{3}:=\sigma \tau\left(M_{0,2,1}\right), & \phi_{4}:=\sigma \tau\left(M_{1,2,0}\right) .
\end{array}
$$

Obviously, $\phi_{l}(l=1, \ldots, 4)$ are in $W^{1,2}$. Our result can be stated in the following

THEOREM. The collection $\Phi$ satisfies (i) ${ }_{1}$ but not (ii) $)_{1}$.

PROOF. To prove the first assertion, we start with the box spline $M_{1,1,1}$. This spline is well known to finite element analysts as the standard linear element, which was first introduced by Courant $[\mathbf{C}]$. The spline $M_{1,1,1}$ has the hexagon $\left\{\lambda_{1} e_{1}+\right.$ $\left.\lambda_{2} e_{2}+\lambda_{3} e_{3} ; 0 \leq \lambda_{i} \leq 1\right\}$ as its support, and $M_{1,1,1}(1,1)=1$. Let $\phi=\sigma \tau\left(M_{1,1,1}\right)$. We claim

$$
\sum_{j \in \mathbf{Z}^{\mathbf{2}}} p(j) \phi(\cdot-j)=p \text { for any } p \in \pi_{1} .
$$

To this end, we recall the following elementary properties of box splines (see $[\mathrm{BH}]$ ):

$$
\sum M_{\Xi}(\cdot-j)=1
$$

and

$$
D_{i}\left(\sum a(j) M_{\Xi}(\cdot-j)\right)=\sum\left(\nabla_{i} a\right)(j) M_{\Xi \backslash e_{i}}(\cdot-j) \quad \text { if } e_{i} \in \Xi .
$$

For $p \in \pi_{1}, D_{i} p=\nabla_{i} p(i=1,2)$ are constants; hence

$$
D_{i}\left(\sum p(j) M_{1,1,1}\left(\cdot-j+e_{3}\right)\right)=\sum\left(\nabla_{i} p\right)(j) M_{\left\{e_{1}, e_{2}, e_{3}\right\} \backslash e_{i}}\left(\cdot-j+e_{3}\right)=D_{i} p .
$$


Moreover,

This shows that

$$
\sum p(j) M_{1,1,1}\left(0-j+e_{3}\right)=p(0) .
$$

$$
\sum p(j)\left(\tau M_{1,1,1}\right)(\cdot-j)=p \quad \text { for } p \in \pi_{1} .
$$

Let the convolution operator $\sigma$ act on both sides of this equality. We have

$$
\sum p(j)\left(\sigma \tau M_{1,1,1}\right)(\cdot-j)=\sigma p .
$$

It is easily seen that $\sigma p=p$ for any $p \in \pi_{1}$. This proves our claim (3).

Now $\{\phi\}$ satisfies hypothesis (ii) $)_{1}$ of Theorem 1 , and (ii) $)_{1}$ implies (i) ${ }_{1}$. Thus for each $u \in W^{2,2}$, we can find $w: \mathbf{Z}^{2} \rightarrow \mathbf{R}$ such that

$$
\left|u-\sum w(j) h^{-1} \phi(\cdot / h-j)\right|_{s, 2} \leq \text { const } h^{2-s}|u|_{2,2}, \quad s=0,1 .
$$

and $\|w\|_{2} \leq$ const $\|u\|_{2}$. The sum in the left-hand side of (5) is denoted by $v$.

We want to express $v$ as an infinite linear combination of $\phi_{i, j}^{h}$. This is possible because of (4). Suppose $\gamma: \mathbf{Z}^{2} \rightarrow \mathbf{R}$ satisfies $\nabla_{1} \gamma=w$. Then it follows from (4) that

$$
\begin{aligned}
\sum & w(j) M_{1,1,1}(\cdot-j)=\sum\left(\nabla_{1} \gamma\right)(j) M_{1,1,1}(\cdot-j) \\
& =D_{1}\left(\sum \gamma(j) M_{2,1,1}(\cdot-j)\right)=\left(D_{3}-D_{2}\right)\left(\sum \gamma(j) M_{2,1,1}(\cdot-j)\right) \\
& =\sum\left(\nabla_{3} \gamma\right)(j) M_{2,1,0}(\cdot-j)-\sum\left(\nabla_{2} \gamma\right)(j) M_{2,0,1}(\cdot-j) .
\end{aligned}
$$

Recall that

$$
\phi=\sigma \tau\left(M_{1,1,1}\right), \quad \phi_{1}=\sigma \tau\left(M_{2,0,1}\right) \quad \text { and } \quad \phi_{2}=\sigma \tau\left(M_{2,1,0}\right) .
$$

We obtain

$$
\sum w(j) \phi_{j}^{h}=\sum\left(\nabla_{3} \gamma\right)(j) \phi_{2, j}^{h}-\sum\left(\nabla_{2} \gamma\right)(j) \phi_{1, j}^{h} .
$$

Thus $v$ can be expressed as an infinite linear combination of $\phi_{1, j}^{h}$ and $\phi_{2, j}^{h}$. But we have trouble in keeping the coefficient sequence $\nabla_{2} \gamma$ bounded in $l^{2}$. To settle this trouble we shall use Fourier series. From now on, the letter $i$ will be reserved for the imaginary unit $\sqrt{-1}$. For any $a \in l^{2}\left(\mathbf{Z}^{2}\right)$, we denote by $\hat{a}$ the Fourier series given by

$$
\hat{a}(\xi):=\sum a(j) e^{2 \pi i j \cdot \xi}, \quad \xi \in \mathbf{R}^{2},
$$

where $j \cdot \xi=j_{1} \xi_{1}+j_{2} \xi_{2}$ is the inner product of $j$ and $\xi$. We have the following elementary property:

$$
\left(\nabla_{k} a\right)^{\Upsilon}=\sum\left(a(j)-a\left(j-e_{k}\right)\right) e^{2 \pi i j \cdot \xi}=\left(1-\exp \left(2 \pi i \xi_{k}\right)\right) \hat{a}, \quad k=1,2 .
$$

Let

$$
g_{k}(\xi):=1-\exp \left(2 \pi i \xi_{k}\right), \quad k=1,2
$$

Then (6) tells us that

$$
\left(\nabla_{2} a\right)^{\widehat{ }}=g_{2} \hat{a}=\left(g_{2} / g_{1}\right)\left(\nabla_{1} a\right) \hat{~}
$$

Let $Q$ be the square $[0,1] \times[0,1]$. By Parseval's identity,

$$
\|\hat{w}\|_{2, Q}:=\left(\int_{Q}|\hat{w}|^{2}\right)^{1 / 2}=\|w\|_{2}
$$


Thus if $\nabla_{1 \gamma}=w$, then

$$
\left\|\nabla_{2} \gamma\right\|_{2}=\left\|\left(\nabla_{2} \gamma\right) \hat{\wedge}\right\|_{2, Q}=\left\|\left(g_{2} / g_{1}\right)\left(\nabla_{1} \gamma\right) \hat{\wedge}\right\|_{2, Q}=\left\|\left(g_{2} / g_{1}\right) \hat{w}\right\|_{2, Q} .
$$

Since $g_{2} / g_{1}$ is unbounded, the norm $\left\|\left(g_{2} / g_{1}\right) \hat{w}\right\|_{2, Q}$ may be unbounded in general. Our scheme is to decompose $w$ into a sum of two sequences $a$ and $b$. For $a$, we seek $\alpha$ such that $\nabla_{1} \alpha=a$ and $\left\|\nabla_{2} \alpha\right\|_{2} \leq\|w\|_{2}$, and for $b$, we find $\beta$ such that $\nabla_{2} \beta=b$ and $\left\|\nabla_{1} \beta\right\|_{2} \leq\|w\|_{2}$. This decomposition of $w$ can be done in the following way: Introduce the set

$$
E:=\left\{\left(\xi_{1}, \xi_{2}\right) \in Q ;\left|g_{1}(\xi)\right| \geq\left|g_{2}(\xi)\right|\right\},
$$

and its characteristic function

$$
\chi_{E}(\xi):= \begin{cases}1 & \text { if } \xi \in E, \\ 0 & \text { if } \xi \in Q \backslash E .\end{cases}
$$

Decompose $\hat{w}$ into $\hat{w} \chi_{E}+\hat{w}\left(1-\chi_{E}\right)$. Obviously,

$$
\left\|\hat{w} \chi_{E}\right\|_{2, Q} \leq\|\hat{w}\|_{2, Q} \text { and }\left\|\hat{w}\left(1-\chi_{E}\right)\right\|_{2, Q} \leq\|\hat{w}\|_{2, Q} .
$$

Hence we can expand $\hat{w} \chi_{E}$ and $\hat{w}\left(1-\chi_{E}\right)$ in Fourier series:

$$
\hat{w} \chi_{E}(\xi)=\sum a(j) e^{2 \pi i j \cdot \xi}, \quad \hat{w}\left(1-\chi_{E}\right)(\xi)=\sum b(j) e^{2 \pi i j \cdot \xi}
$$

with $\|a\|_{2},\|b\|_{2} \leq\|\hat{w}\|_{2, Q}=\|w\|_{2}$. It follows that

$$
\sum w(j) e^{2 \pi i j \cdot \xi}=\hat{w}(\xi)=\sum(a(j)+b(j)) e^{2 \pi i j \cdot \xi} .
$$

By the uniqueness of Fourier series expansion, we have $w=a+b$. Since $\hat{a}=\hat{w} \chi_{E}$, and since $\left|g_{2}(\xi)\right| \leq\left|g_{1}(\xi)\right|$ for $\xi \in E$, we have

$$
\left|\left(g_{2} / g_{1}\right) \hat{a}(\xi)\right| \leq|\hat{a}(\xi)| \text { for } \xi \in Q,
$$

and therefore we can find $c \in l^{2}\left(\mathbf{Z}^{2}\right)$ such that

$$
\hat{c}=\left(g_{2} / g_{1}\right) \hat{a} \text {. }
$$

In addition,

$$
\|c\|_{2}=\|\hat{c}\|_{2, Q} \leq\|\hat{a}\|_{2, Q}=\|a\|_{2} \leq\|w\|_{2} .
$$

Furthermore, (8) is equivalent to

$$
g_{1} \hat{c}=g_{2} \hat{a}, \quad \text { or } \quad\left(\nabla_{1} c\right) \hat{-}=\left(\nabla_{2} a\right) \hat{;}
$$

hence $\nabla_{1} c=\nabla_{2} a$.

Having obtained $a$ and $c$, we can find (uniquely) a sequence $\alpha: \mathbf{Z}^{2} \rightarrow \mathbf{R}$ such that $\nabla_{1} \alpha=a$ and

$$
\alpha\left(0, j_{2}\right)= \begin{cases}\sum_{l=1}^{j_{2}} c(0, l) & \text { for } j_{2}>0 \\ 0 & \text { for } j_{2}=0 \\ -\sum_{l=j_{2}+1}^{0} c(0, l) & \text { for } j_{2}<0\end{cases}
$$

Then

$$
\nabla_{1}\left(\nabla_{2} \alpha\right)=\nabla_{2}\left(\nabla_{1} \alpha\right)=\nabla_{2} a=\nabla_{1} c
$$

But (9) tells us that

$$
\nabla_{2} \alpha\left(0, j_{2}\right)=c\left(0, j_{2}\right) \quad \text { for all } j_{2} \in \mathbf{Z}
$$


therefore $\nabla_{2} \alpha=c$. Furthermore

$$
\begin{aligned}
\nabla_{3} \alpha & =\alpha-\alpha\left(\cdot-e_{3}\right)=\alpha-\alpha\left(\cdot-e_{2}\right)+\alpha\left(\cdot-e_{2}\right)-\alpha\left(\cdot-e_{3}\right) \\
& =\nabla_{2} \alpha+\nabla_{1} \alpha\left(\cdot-e_{2}\right)=\nabla_{2} \alpha+a\left(\cdot-e_{2}\right)
\end{aligned}
$$

hence

$$
\left\|\nabla_{3} \alpha\right\|_{2} \leq 2\|a\|_{2} \leq 2\|w\|_{2} .
$$

To sum up, we can find $\alpha: \mathbf{Z}^{2} \rightarrow \mathbf{R}$ such that

$$
\left\|\nabla_{2} \alpha\right\|_{2} \leq\|w\|_{2}, \quad\left\|\nabla_{3} \alpha\right\|_{2} \leq 2\|w\|_{2}
$$

and

$$
\sum a(j) \phi_{j}^{h}=\sum\left(\nabla_{3} \alpha\right)(j) \phi_{2, j}^{h}-\sum\left(\nabla_{2} \alpha\right)(j) \phi_{1, j}^{h} .
$$

Similarly, we can find $\beta: \mathbf{Z}^{2} \rightarrow \mathbf{R}$ such that

$$
\left\|\nabla_{1} \beta\right\|_{2} \leq\|w\|_{2}, \quad\left\|\nabla_{3} \beta\right\|_{2} \leq 2\|w\|_{2}
$$

and

Recall that

$$
\sum b(j) \phi_{j}^{h}=\sum\left(\nabla_{3} \beta\right)(j) \phi_{4, j}^{h}-\sum\left(\nabla_{1} \beta\right)(j) \phi_{3, j}^{h} .
$$

From (5) we observe that

$$
v=\sum w(j) \phi_{j}^{h}=\sum a(j) \phi_{j}^{h}+\sum b(j) \phi_{j}^{h}
$$

$$
|u-v|_{s, 2} \leq \text { const } h^{2-s}|u|_{2,2}, \quad s=0,1 .
$$

Thus we have proved that the collection $\Phi=\left\{\phi_{1}, \phi_{2}, \phi_{3}, \phi_{4}\right\}$ satisfies (i) .

It remains to show that $\Phi$ does not satisfy (ii) $)_{1}$. This will be done by contradiction. Suppose to the contrary that there is a finite linear combination $\Omega$ of $\phi_{1}, \ldots, \phi_{4}$ and their translates $\phi_{l, j}$ such that

for $|\alpha| \leq 1, \sum_{j \in \mathbf{Z}^{2}} j^{\alpha} \Omega(x-j)$ is a polynomial in $x_{1}, x_{2}$ with leading term $x^{\alpha}$.

Assume

$$
\Omega=\sum_{l=1}^{4} \sum_{m \in I} c_{l, m} \phi_{l}(\cdot-m)
$$

where $I$ is a finite subset of $\mathbf{Z}^{2}$. We want to draw a contradition from the above assumption. Let us first take a closer look at the function $\phi_{1}$. By straightforward computation we have

$$
\phi_{1}\left(x_{1}, x_{2}\right)=M_{2}\left(x_{1}-x_{2}+1\right) \psi\left(x_{2}\right)
$$

with

$$
\psi\left(x_{2}\right)= \begin{cases}2\left(x_{2}+5 / 4\right) & \text { for }-5 / 4 \leq x_{2} \leq-3 / 4 \\ 1 & \text { for }-3 / 4 \leq x_{2} \leq-1 / 4 \\ -2\left(x_{2}-1 / 4\right) & \text { for }-1 / 4 \leq x_{2} \leq 1 / 4 \\ 0 & \text { for } x_{2}<-4 / 5 \text { or } x_{2}>1 / 4\end{cases}
$$

This motivates us to introduce the following functional $F$ :

$$
F g:=\lim _{\varepsilon \rightarrow+0}\left[D_{3} g\left(\frac{1}{4}+\varepsilon, \frac{1}{4}+\varepsilon\right)-D_{3} g\left(\frac{1}{4}-\varepsilon, \frac{1}{4}-\varepsilon\right)\right] \quad \text { for } g \in W^{1, \infty}\left(\mathbf{R}^{2}\right) \text {. }
$$


Just by computation, we obtain

It follows that

$$
F\left(\phi_{1}(\cdot-j)\right)= \begin{cases}2 & \text { if } j=(0,0) \\ -2 & \text { if } j=(1,1) \\ 0 & \text { otherwise }\end{cases}
$$

By (4) we have

$$
F\left(\sum j_{2} \phi_{1}(\cdot-j)\right)=-2
$$

$$
\begin{aligned}
\sum j_{2} M_{2,1,0}(\cdot-j) & =D_{3} \sum\left(j_{2}\left(j_{2}+1\right) / 2\right) M_{2,1,1}(\cdot-j) \\
& =\left(D_{1}+D_{2}\right) \sum\left(j_{2}\left(j_{2}+1\right) / 2\right) M_{2,1,1}(\cdot-j) \\
& =\sum j_{2} M_{2,0,1}(\cdot-j)
\end{aligned}
$$

hence

$$
F\left(\sum j_{2} \phi_{2}(\cdot-j)\right)=F\left(\sum j_{2} \phi_{1}(\cdot-j)\right)=-2 .
$$

By an argument similar to that used in proving (3), we observe that

$$
\sum_{j} j_{2} \phi_{l}(\cdot-j) \text { is in } \pi_{1} \text { for } l=3 \text { or } 4 \text {. }
$$

Therefore

$$
F\left(\sum_{j} j_{2} \phi_{l}(\cdot-j)\right)=0, \quad l=3 \text { or } 4 .
$$

Now we consider the function $q:=\sum j_{2} \Omega(\cdot-j)$. On the one hand, $q \in \pi_{1}$; hence $F(q)=0$. On the other hand, by $(10)$,

$$
q=\sum_{j} j_{2} \Omega(\cdot-j)=\sum_{j} \sum_{m} \sum_{l=1}^{4} c_{l, m} j_{2} \phi_{l}(\cdot-m-j),
$$

hence by (11) and (12),

$$
F(q)=\sum_{m} \sum_{l=1}^{4} c_{l, m} F\left(\sum j_{2} \phi_{l}(\cdot-m-j)\right)=-2 \sum_{l=1}^{2} \sum_{m} c_{l, m} .
$$

This shows that

Similarly, one can show

$$
\sum_{l=1}^{2} \sum_{m} c_{l, m}=0
$$

$$
\sum_{l=3}^{4} \sum_{m} c_{l, m}=0
$$

Finally, since $\sum \phi_{l}(\cdot-j)=1$, we get

$$
\sum \Omega(\cdot-j)=\sum_{l=1}^{4} \sum_{m} c_{l, m}\left(\sum_{j} \phi_{l}(\cdot-m-j)\right)=\sum_{l=1}^{4} \sum_{m} c_{l, m}=0 .
$$

This shows that $\Phi$ does not satisfy (ii) ${ }_{1}$. Our proof is complete. 


\section{REFERENCES}

[BH] C. De Boor and K. Höllig, B-splines from parallelepipeds, J. Analyse Math. 42 (1983), 99-115.

[C] R. Courant, Variational method for the solution of problems of equilibrium and vibrations, Bull. Amer. Math. Soc. 49 (1943), 1-33.

[DM] W. Dahmen and C. A. Micchelli, On the approximation order from certain multivariate spline spaces, J. Austral. Math. Soc. Ser. B 26 (1984), 233-246.

[FS] G. Fix and G. Strang, Fourier analysis of the finite element method in Ritz-Galerkin theory, Stud. Appl. Math. 48 (1969), 265-273.

[S] G. Strang, The finite element method and approximation theory, Numerical Solution of Partial Differential Equations (B. Hubbard, ed.), SYNSPADE 1970, Univ. of Maryland, College Park, 1970, pp. 547-583.

[SF] G. Strang and G. Fix, A Fourier analysis of the finite element variational method, Constructive Aspects of Functional Analysis (G. Geymonat, ed.) (C.I.M.E. II, Ciclo Erice, 1971), 1973, pp. 793-840.

Mathematics Research Center, University of Wisconsin, MAdison, WisconSIN 53705

Current address: Department of Mathematics, Zhejiang University, Hangzhou, Zhejiang, The People's Republic of China 